6-1-2012

\title{
Periprosthetic joint infection: are patients with multiple prosthetic joints at risk?
}

S Mehdi Jafari

The Rothman Institute of Orthopaedics, Thomas Jefferson University Hospital

David S Casper

The Rothman Institute of Orthopaedics, Thomas Jefferson University Hospital

Camilo Restrepo

The Rothman Institute of Orthopaedics, Thomas Jefferson University Hospital

Benjamin Zmistowski

The Rothman Institute of Orthopaedics, Thomas Jefferson University Hospital

Javad Parvizi

The Rothman Institute of Orthopaedics, Thomas Jefferson University Hospital Follow this and additional works at: https://jdc.jefferson.edu/rothman_institute

Part of the Orthopedics Commons

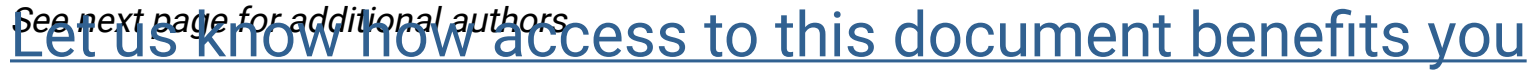

\section{Recommended Citation}

Jafari, S Mehdi; Casper, David S; Restrepo, Camilo; Zmistowski, Benjamin; Parvizi, Javad; and Sharkey, Peter F, "Periprosthetic joint infection: are patients with multiple prosthetic joints at risk?" (2012). Rothman Institute Faculty Papers. Paper 16.

https://jdc.jefferson.edu/rothman_institute/16

This Article is brought to you for free and open access by the Jefferson Digital Commons. The Jefferson Digital Commons is a service of Thomas Jefferson University's Center for Teaching and Learning (CTL). The Commons is a showcase for Jefferson books and journals, peer-reviewed scholarly publications, unique historical collections from the University archives, and teaching tools. The Jefferson Digital Commons allows researchers and interested readers anywhere in the world to learn about and keep up to date with Jefferson scholarship. This article has been accepted for inclusion in Rothman Institute Faculty Papers by an authorized administrator of the Jefferson Digital Commons. For more information, please contact: JeffersonDigitalCommons@jefferson.edu. 
Authors

S Mehdi Jafari, David S Casper, Camilo Restrepo, Benjamin Zmistowski, Javad Parvizi, and Peter F Sharkey 


\section{As submitted to:}

The Journal of arthroplasty
And later published as:

Periprosthetic Joint Infection: Are Patients with Multiple

Prosthetic Joints at Risk?

Volume 27, Issue 6, June 2012, Pages 877-880

DOI: 10.1016/j.arth.2012.01.002

Jafari, S.M., Casper, D.S., Restrepo, C., Zmistowski, B., Parvizi, J., Sharkey, P.F.

The Rothman Institute of Orthopaedics, Thomas Jefferson University Hospital, Philadelphia, PA, United States 
Abstract

Patients that present with a periprosthetic joint infection in a single joint may have multiple prosthetic joints. The risk of these patients developing a subsequent infection in another prosthetic joint is unknown. Our purposes were (1) identify the risk of developing a subsequent infection in another prosthetic joint; and (2) describe the time span, and organism profile to the second prosthetic infection. We retrospectively identified 55 patients with periprosthetic joint infection who had another prosthetic joint in place at the time of presentation. Eleven of the 55 patients (20\%) developed a periprosthetic joint infection in a second joint. The type of organism was the same as the first infection in four of 11 patients (36\%). The time to developing a second infection was 2.0 years (range, 0 6.9 years).

Keywords: Multiple Periprosthetic Joint Infection; Hip Arthroplasty; Knee Arthroplasty; Risk of subsequent infection; Phenotypically Identical Infecting Organisms. 
Introduction

Periprosthetic joint infection (PJI) is an important failure mechanism of implants after either primary or revision total joint arthroplasty $(T J A)^{1-3}$. It occurs in approximately $0.2 \%$ to $2.5 \%$ of primary THAs or TKAs ${ }^{2,4-6}$. Revision THA or TKA carries an infection risk of $3.2 \%$ to $9 \%^{2,3}$. When PJI develops, an extensive burden is placed on the affected patient and the treating physician. PJI causes considerable increase in the cost of health care and increases the rate of morbidity and mortality ${ }^{2,7}$.

On occasion, patients presenting with developed PJI have multiple lower limb prosthetic joints. It is always a concern that the presence of any nidus of ongoing infection in the body can lead to PJI. Therefore, it is cause for concern that patients may develop PJI in coexisting, aseptic joints when another joint becomes infected. To mediate infections, surgeons use methods such as antibiotics, irrigation and debridement, and antibiotic spacers. In order to help curtail infection, patients are encouraged to take antibiotics before procedures that are considered high risk for inducing bacteremia, such as dental procedures. However, the role of these prophylactic antibiotics is a matter of controversy $y^{8,9}$.

Therefore, we had two objectives: (1) to identify the risk of developing an additional PJI in a coexisting prosthetic joint; and (2) to describe the time to additional infection and organism profile of the second prosthetic infection. 
Patients and Methods

We used our joint infection database to identify patients that had confirmed diagnoses of PJI at our institute from 2000 to 2009. These patients were crossreferenced with our prospective joint arthroplasty database to identify all patients who had at least two primary arthroplasties (hip or knee) performed at this institution and later developed a PJI in at least one. We identified 55 patients. These patients averaged 63.4 years of age (range: $31-90$ years) and 26 of the 55 (47\%) were male. The average BMI of these 55 patients was $32.2 \mathrm{~kg} / \mathrm{m}^{2}(95 \% \mathrm{CI}$ : 30.1-34.2). Out of the 55 patients, 46 (84\%) presented with pertinent comorbidities (Table 1). Seventeen (30.9\%; 17/55\%) patients developed a PJI within a four week period postoperatively, thus indicating an acute onset. The remaining $38(\%)$ developed their first incident of PJI within 8.2 years. Nine (16\%) of these patients presented with ipsilateral implants, 37 (67\%) were contralateral knees, 16 (29\%) were contralateral hips, and nine (16\%) were contralateral hip and knee. Five patients had three prosthetic joints and two patients had both hips and both knees replaced. The total number of joints does not total 55 because of patients who presented with more than two joints replaced. These patients with three and four joints replaced constitute the discrepancy of the overall figure and the 55 patients noted (Table 1). Infection was defined as the 
presence of a positive preoperative fluid or intraoperative tissue culture, presence of purulence, or elevated erythrocyte sedimentation rate, $C$-reactive protein, synovial white blood cell, and polymorphonuclear percentage $e^{10}$. Follow-up for this study is defined as patients that suffered a single PJI while having multiple TJA in place, who subsequently returned for an additional infection to a second joint. In this case no patients were lost to follow-up; however, it is possible that patients retained services for an additional infection elsewhere. No patients were recalled specifically for this study; all data was obtained from medical records. We had prior approval of our Institutional Review Board.

To ascertain the rate of infection in a prosthetic joint after development of PJI in the first joint, the joint infection database at this institution was again used to identify any subsequent infections in these patients. It was verified that the subsequent infection cases developed in prosthetic joints that had been replaced before the development of the first periprosthetic joint infections.

The nature of the second infection was analyzed by noting the duration between the initial infection and the subsequent infection, and determining the organism profile for each infection. Organisms of the two infections were considered identical if they were of identical species and had identical resistance profiles. 
To ascertain potential predictors of suffering multiple joint PJI in this group of patients, univariate statistical analysis was performed on potential variables (Table 1). This included age, BMI, gender, systemic health, and status of joint replacements. Systemic health was measured with the Deyo et al modification of the Charlson Index. ${ }^{11}$ A review of charts was also performed in attempts to identify specific comorbidities that may contribute to risk of subsequent additional joint PJI. Statistical analysis consisted of a Student's t-test for continuous variables and Chi-squared test for categorical variables. Analysis was performed using PASW Statistics 18.0.0 (SPSS Incorporated, Chicago, IL).

Results

Of the 55 patients who presented with multiple coexisting prosthetic joints, with PJI present in at least one, 11 patients (20\%) subsequently developed PJI in a second coexisting prosthetic joint. The second joint infection developed in contralateral knees in eight (73\%) of the 11 patients, contralateral hips in two (18\%) of the 11 patients, and ipsilateral hip and knee in one patient (9\%) (Figure 1). The average BMI of these 11 patients was found to be $31.3 \mathrm{~kg} / \mathrm{m}^{2}$, demonstrating no statistical difference when compared to that of the overall cohort. When comparing specific comorbidities of the patients diagnosed with multiple infections versus those with a solitary infection no statistical difference exists. However, when comparing the multiple versus single PJI groups, the 
multiple PJI group had a greater median Charlson Index (4 versus 2.5; $p=0.16$ ). As well, the initial PJI incident was less likely to have occurred in the acute postoperative setting (less than four weeks) compared to the single PJI group (9.1\% versus $36.4 \% ; p=0.08)$

Of these 11 patients that sustained multiple PJIs, four were diagnosed as having phenotypically identical organisms for both infections. For two of these patients, the presentation of PJI was simultaneous. The two remaining patients presented with the second joint infection at 4 months and 3.5 years, respectively (Figure 2). The time from presentation of the original PJI to the infection in the second joint averaged 2.0 years (range, $0-6.88$ years).

\section{Discussion}

Several studies have been performed to find the risk factors for infection after TJA to decrease the rate of this devastating complication ${ }^{6,8,9,12,13}$. Accordingly, several preventive measures have been recommended to decrease the rate of $\mathrm{PJI}^{8,9}$. However, the authors were unable to find any literature that addresses the risk of developing synchronous or metachronous PJI. Therefore, we had two objectives: (1) to identify the risk of developing an additional PJI in a coexisting prosthetic joint; and (2) to describe the time to additional infection and organism profile of the second prosthetic infection. 
Our study has some limitations. First, this investigation is a retrospective review of a database; the data is input by clerks and as such is subject to clerical error and/or may be incomplete, rendering a lower yield of complete data set. Second, despite all efforts to capture all PJI in our institution during the study time, some patients may have sought medical attention at other institutions in the event of developing PJI. Finally, despite evaluating all data referring to diagnostic workup for PJI, some origin or nidus of infection that could have led to a subsequent PJI may have been missed. This would yield different results in the mechanism of infection, which is why we refrain ourselves from establishing such in the article.

We found that 55 patients presented with multiple coexisting prosthetic joints, in which at least one developed PJI. Eleven patients developed a PJI in a second coexisting prosthetic joint. Therefore, the relative risk of developing a synchronic or metachronic PJI is $20 \%$. In patients who did not present with simultaneous PJI, the time of presentation for metachronic PJI averaged 2.71 years.

This increased risk of developing another PJI appears excessively high compared even with the highest incidence of isolated PJI reported in the literature. In our cohort, all patients shared the same preoperative, intraoperative, and postoperative protocols (prophylactic antibiotic, laminar flow, personal protection 
system, etc), similar hospital stay, and same postoperative rehabilitation regimen. The fact that some patients with multiple TJA only develop an isolated PJI and some develop multiple PJI may indicate a host compromise or a reoccurring exposure despite the similarities in preexisting condition and demographics between the multiple and single PJI groups. While statistical significance was not achieved, the large difference in Charlson Index supports the reasoning that patients who developed multiple PJI have decreased general health contributing to multiple joint PJI. Supporting this is the observation that a third of single PJI patients developed PJI within the acute postoperative period, while less than $10 \%$ of multiple PJI patients developed PJI in the acute postoperative period. This points to a compromised immune system and lower overall health leading to PJI in the multiple PJI group while surgical contamination appears to be a major factor in the single PJI group. This would suggest that the risk of multiple infections do not coincide with presence of multiple joints but rather the associated traits and actions of the patient.

Four of the 11 patients (36\%) with multiple PJI were diagnosed as having phenotypically identical infecting organisms for both joints (Figure 1); in two of these patients, the presentation was synchronic, suggesting a hematogenous mode of infection. In the other two metachronic PJI, host characteristics were most likely 
the cause of multiple infections. In all four of these patients, the involved joints where contralateral.

This evaluation shows that there is a high risk of developing multiple joint PJI when patients present with PJI in a single joint and multiple prosthetic joints. It appears that the risk is elevated in immunocompromised patients suffering chronic or delayed PJI. Future research is needed to confirm the risk factors identified here and develop methods for minimizing the risk of multiple joint PJI. Regardless of the cause, it is evident that there is a significant risk of multiple joint PJI (20\%) and therefore, the surgeon should cautiously investigate any asymptomatic joints in the face of a single PJI, and alert the patient to the increased risk of subsequent PJI. 


\section{$1 \quad$ References}

1. Jafari SM, Coyle C, Mortazavi SMJ, Sharkey PF, Parvizi J. Revision hip arthroplasty: infection is the most common cause of failure. Clin. Orthop. Relat. Res. 2010;468(8):2046-2051.

2. Lentino JR. Prosthetic joint infections: bane of orthopedists, challenge for infectious disease specialists. Clin. Infect. Dis. 2003;36(9):1157-1161.

3. Mortazavi SMJ, Schwartzenberger J, Austin MS, Purtill JJ, Parvizi J. Revision total knee arthroplasty infection: incidence and predictors. Clin. Orthop. Relat. Res. 2010;468(8):2052-2059.

10 4. Kurtz S, Ong K, Lau E, et al. Prosthetic Joint Infection Risk after TKA in the 11 Medicare Population. Clin. Orthop. Relat. Res. 2009. Available at:

12 http://www.ncbi.nlm.nih.gov/pubmed/19669386. Accessed November 30, 2009.

13 5. Ong KL, Kurtz SM, Lau E, et al. Prosthetic joint infection risk after total hip 14 arthroplasty in the Medicare population. J Arthroplasty. 2009;24(6 Suppl):10515109.

16 6. Urquhart DM, Hanna FS, Brennan SL, et al. Incidence and risk factors for deep 17 surgical site infection after primary total hip arthroplasty: a systematic review. J 18 Arthroplasty. 2010;25(8):1216-1222.e1-3.

19 7. Parvizi J, Pawasarat IM, Azzam KA, et al. Periprosthetic joint infection: the 20 economic impact of methicillin-resistant infections. J Arthroplasty. 2010;25(6 21 Suppl):103-107.

22 8. Berbari EF, Osmon DR, Carr A, et al. Dental Procedures as Risk Factors for 23 Prosthetic Hip or Knee Infection: A Hospital-Based Prospective Case-Control 24 Study. Clin. Infect. Dis. 2009. Available at:

25 http://www.ncbi.nlm.nih.gov/pubmed/19951109. Accessed December 7, 2009.

26 9. Uçkay I, Pittet D, Bernard L, et al. Antibiotic prophylaxis before invasive dental 27 procedures in patients with arthroplasties of the hip and knee. J Bone Joint Surg 28 Br. 2008;90(7):833-838.

29 10. Parvizi J, Jacovides C, Zmistowski B, Jung KA. Definition of Periprosthetic 30 Joint Infection: Is There a Consensus? Clin Orthop Relat Res. 2011. Available at: 31 http://www.ncbi.nlm.nih.gov/pubmed/21751038. Accessed July 14, 2011. 
32 11. Deyo RA, Cherkin DC, Ciol MA. Adapting a clinical comorbidity index for use

33 with ICD-9-CM administrative databases. J Clin Epidemiol. 1992;45(6):613-619.

34 12. Kurtz SM, Ong KL, Lau E, et al. Prosthetic Joint Infection Risk after TKA in

35 the Medicare Population. Clin Orthop Relat Res. 2010;468(1):52-56.

36 13. Pulido L, Ghanem E, Joshi A, Purtill JJ, Parvizi J. Periprosthetic joint

37 infection: the incidence, timing, and predisposing factors. Clin. Orthop. Relat. Res.

38 2008;466(7):1710-1715.

39 